http://dx.doi.org/10.21611/qirt.1992.048

\title{
Infrared measurements of electromagnetic fields
}

\author{
by J. NORGARD $\left(^{\star}\right)$, D. METZGER(*), R. SEGA $\left(^{\star}\right)$, M. HARRISON(**), \\ R. KOMAR( $\left(^{\star \star}\right)$, H. POHLE $\left({ }^{* *}\right)$, A. SCHMELZEL $\left({ }^{* *}\right)$, M. SMITH( $\left.{ }^{\star *}\right)$, \\ J. STUPIC $\left({ }^{\star \star}\right)$, M. SEIFERT $\left(^{\star \star \star}\right)$ and J. CLEARY $\left({ }^{* \star}\right)$
}

(*) Electromagnetics Laboratory, University of Colorado/Colorado Springs, Colorado, USA.

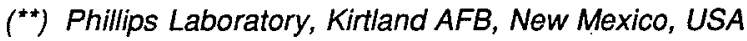

$\left(^{* * *}\right)$ Rome Laboratory, Griffiss AFB, New York, USA.

\begin{abstract}
In this paper, an infrared (IR) measurement technique is presented which has been developed to measure electromagnetic (EM) fields. This technique uses a minimally perturbing planar IR detection screen to map the intensity of the EM energy over a two-dimensional region. Several examples of measured EM fields near radiating sources and scattering bodies using IR thermograms are presented.
\end{abstract}

\section{Introduction}

A non-destructive, minimally perturbing infrared (IR) measurement technique has been developed to observe electromagnetic (EM) fields. Metallic surface currents and charges also can be measured with this technique.

This IR measurement technique produces a two-dimensional IR thermogram of the electric or magnetic field being measured, i.e. an isothermal contour map of the intensity of the EM field.

This IR measurement technique recently has been applied to determine microwave induced surface currents [1], radiated fields from antennas and high power microwave (HPM) sources, e.g. near-field intensities and far-field antenna patterns of horns [2-3], diffraction patterns of EM fields scattered from complicated metallic objects [4-7], intensities of EM fields coupled through apertures in shielded enclosures [8-11], and modal distributions of EM fields excited inside cavities [12-15].

Examples of EM energy radiated from a horn antenna, coupling through an aperture, and induced cylindrical cavity modes are presented in this paper. The advantages and disadvantages of this new IR measurement technique are also discussed.

\section{IR measurement technique}

The IR measurement technique is based on the Joule heating that occurs in a lossy material as an EM wave passes through the material. A thin planar sheet of a lossy carbon loaded material is used to map electric fields; a thin planar sheet of a lossy ferrite loaded material is used to map magnetic fields. The absorbed heat energy is converted into conducted and convected heat energy and into re-radiated EM energy. The radiated EM energy is concentrated in the IR band, and is detected with an IR Scanning Array or an IR Focal Plane Array (FPA) as the energy radiated from a black body. 


\subsection{IR experimental setup}

This technique involves placing the lossy IR detection screen near the EM source in the plane over which the fields are to be measured. The loss mechanisms in the detector material, i.e. the conductivity and the imaginary components of the permittivity and the permeability of the detector material, absorb the incident EM energy as thermal heat energy and cause the temperature of the detector to rise above the ambient temperature of the surrounding environment by an amount that is proportional to the local electric and/or magnetic field intensity (energy) at each point in the screen material. This produces a two-dimensional IR thermogram of the electric and/or magnetic field in the screen material.

\subsection{IR detector screen}

The screen material is tailored to be sensitive to only one component of the field, e.g. by optimizing the values of the electrical conductivity and the imaginary part of the permittivity of the material relative to the imaginary part of the permeability of the material, the detector screen can be made sensitive to only the tangential component of the electric or magnetic field in the plane of the screen. Care is also exercised in the selection of the screen material not to significantly perturb the electric or magnetic field by the presence of the lossy material. The screen is designed to absorb less than $5 \%$ of the incident power. The constitutive parameters of the IR detector screen are optimized to produce a large temperature rise in the detector material for a small amount of absorbed energy.

The temperature difference between the screen material and the background is detected, digitized, and stored in the memory of the IR camera on a pixel by pixel basis. The system has 128 by 128 pixels per frame of data. This stored data represents the temperature distribution over the extent of the detector screen and is a map of the intensity of the electric or magnetic field distribution absorbed in the screen. The incident electric or magnetic field can then be determined from a solution of Maxwell's equations (Fresnel's and Snell's laws) for an EM wave incident on a planar film of lossy material. For small temperature rises, the electric or magnetic field intensity is nearly linearly proportional to the temperature change.

\subsection{IR measurement accuracy}

The accuracy of the IR measurement technique can be demonstrated by performing two simple experiments with known theoretical solutions. In one experiment, as shown in figure 1, the diffraction pattern from a Lloyd's mirror was measured. In the other experiment, as shown in figure 2, the diffraction pattern from an knife edge conducting half-plane was measured. In the Lloyd's mirror experiment, the resulting diffraction pattern is due to the antenna interfering with its image in the ground plane. In both experiments, the screen material was optimized to measure only the tangential component of the electric field intensity in the plane of the screen. The screen material was made from a thin planar sheet of carbon paper (Teledeltos Paper) $80 \mu \mathrm{m}$ thick with a conductivity of 8 mhos per meter and negligibly small imaginary permittivity and permeability. These experiments were performed in an anechoic chamber, as shown in figures $1 a$ and $2 a$. Good correlations between theory and experiment were obtained, as shown in figures $1 b$ and $2 b$. The worst errors occurred in the minimums (deep nulls) of the diffraction patterns were thermal bleeding from the surrounding hot areas tended to obscure the real depth of the minimums. Some thermal bleeding out of the maximums into the surrounding areas also occurred, obscuring the real height of the maximums.

\subsection{IR advantages and disadvantages}

The IR measurement technique provides a quick and accurate method to observe EM fields in a two-dimensional plane. However, only the magnitude of the electric or magnetic field is measured; no phase information is detected. Also, since this technique is based on the thermal mass of a detection material, high energy is required to produce good thermal images of EM fields. 


\section{http://dx.doi.org/10.21611/qirt.1992.048}

\section{Scattering from and coupling into a cylinder}

As an example of the IR measurement technique, a right circular cylinder, containing a long, thin slot aperture in its side, was irradiated with a plane EM wave. IR thermograms were made of the diffraction pattern of the EM field scattered from the cylinder and the cylindrical modes excited inside the cavity. The experimental setup is shown in figures $3 a$ and $3 b$.

The cylinder is $1 \mathrm{~m}$ in length and has an inner diameter of approximately $10 \mathrm{~cm}$. The rectangular slot aperture is $64 \mathrm{~mm}$ in width and is $10 \mathrm{~cm}$ in length. The slot is located in the middle of the cylinder and is oriented parallel to the axis of the cylinder. The cylinder is irradiated with microwave energy at $3 \mathrm{GHz}(10 \mathrm{~cm}$ wavelength) from a pyramidal horn antenna, polarized in the circumferential direction of the cylinder. Cylindrical TE modes are predominantly excited inside the cavity.

A large IR detector screen of carbon paper was positioned in the radial plane that intersected the middle of the slot aperture. Another IR disk was positioned inside the cylinder in the same cross-sectional plane.

Experimentally obtained IR thermograms of the results are show in figures $A, B, C$. The brightness of each color in the image corresponds to the intensity of the EM field. Thermograms of the incident microwave field of the horn antenna are shown in figure $A^{*}$. Figure $A a$ is an image of the electric field traveling wave in the longitudinal plane in front of the horn. Figure $A b$ is an image of the electric field in the transverse plane one meter in front of the aperture of the horn. Thermograms of the field scattered from the cylinder are shown in figure $B$. Figure $B a$ is an image of the electric field standing wave created between the horn and the cylinder. This thermogram shows the interference pattern between the incident wave and the scattered cylindrical wave. Figure $B b$ is an image of the electric field travelling wave in the shadow zone of the cylinder. The null behind the cylinder and the diffracted wave off the top and bottom of the cylinder can be seen in this thermogram. Thermograms of the induced modes coupled into the cylindrical cavity are shown in figure $\mathrm{C}$. Figure $\mathrm{Ca}$ is an image of the electrical field coupled through the aperture for a frequency slightly below the cutoff frequency of the cylindrical waveguide. The EM energy coupled through the aperture is visible in this thermogram and, as expected, has the radiation pattern of an electric dipole. The dominant TE,I waveguide modal pattern is partially developed in the center of the waveguide. Figure $\mathrm{Cb}$ is an image of the electrical field coupled through the aperture for a frequency slightly above the cutoff frequency of the cylindrical waveguide. The dominant TE1, waveguide modal pattern is now fully developed in the center of the waveguide.

The diffraction patterns of the EM fields scattered from the cylinder and the modal patterns of the induced cylindrical cavity modes are clearly indicated in these figures.

\section{Conclusion}

The IR measurement technique is a viable method to aid in the determination of EM fields scattered from complex metallic objects and the EM energy coupled into complex cavity structures. The IR method allows for rapid observation of EM field activity and interference, resulting in an in-depth understanding of the EM scattering and coupling phenomena.

\section{REFERENCES}

[1] WETLAUFER (G.D.), SEGA (R.M.) and NORGARD (J.D.). - Thermographic detection of microwave induced surface currents. Proceedings of the APS/URSI Symposium, May 1985, Vancouver, BC, Canada.

[2] SEGA (R.M.) and NORGARD (J.D.). - Infrared diagnostic techniques for high-power microwave measurements. Proceedings of High Power Microwave Technology Meeting, December 1986, Albuquerque, NM.

[3] METZGER (D.W.), NORGARD (J.D.) and SEGA (R.M.). - Numerical calculation \& 
experimental verification of near fields from horns. IEEE-EMC Transactions, February 1992. [4] SEGA (R.M.) and NORGARD (J.D.). - Infrared detection of microwave scattering from cylindrical structures. Proceedings of the URSI Winter Meeting, January 1986, Boulder, Co.

[5] SEGA (R.M.) and NORGARD (J.D.). - Infrared measurement of scattering and electromagnetic penetrations through apertures. IEEE-NS Transactions, Vol. NS-33, $N^{\circ} 6$, December 1986, p. 1858-1 883.

[6] SEGA (R.M.), NORGARD (J.D.) and HARRISON (M.G.). - Infrared comparisons of the electromagnetic scattering from conducting and dielectric cylinders. Proceedings of the Nuclear Electromagnetics Symposium, May 1988, San Francisco, Ca.

[7] NORGARD (J.D.), SEGA (R.M.), IANACONE (K.J.), HARRISON (M.G.), PESTA (A.T.) and SEIFERT (M.A.). - Scattering effects of electric and magnetic field probes. IEEE-NS Transactions, Vol. NS-36, N6, December 1989, p. 2050-2057.

[8] SEGA (R.M.) and NORGARD (J.D.). -An infrared measurement technique for the assessment of electromagnetic coupling. IEEE-NS Transactions, Vol. $32, \mathrm{~N}^{\circ} 6$, December 1985, p. 43304332.

[9] METZGER (D.W.), SEGA (R.M.), NORGARD (J.D.) and BUSSEY (P.A.).- Experimental and theoretical techniques for determining coupling through apertures in cylinders. Proceedings of the Nuclear Electromagnetic Meeting, May 1986, Albuquerque, NM.

[10] SEGA (R.M.) and NORGARD (J.D.). - Expansion of an $M$ detection technique using conduchve mesh in microwave shielding applications. Proceedings of the SPIE Symposium, August, 1987, San Diego, Ca.

[11 ] SMITH (M.D.), SEGA (R.M.) and NORGARD (J.D.). - Infrared detection of electromagnetic penetration through narrow slots in a planar conducting surface. Proceedings of the URSI National Radio Science Meeting, January 1990, Boulder, Co.

[12] NORGARD (J.D.) and SEGA (R.M.). - Three-dimensional determination of cavityresonance and internal coupling. Proceedings of the URSI Winter Meeting, January, 1987, Boulder, Co.

[ 13] SEGA (R.M.), NORGARD (J.D.) and GENELLO (G.J.). - Measured internal coupled electromagnetic fields related to cavity and aperture resonance. IEEE-NS Transactions, Vol. NS-34, No 6, December 1987, p. 1502-1507.

[14] NORGARD (J.D.) and SEGA (R.M.). - Measured and predicted coupling of electromagnetic radiation into a cylindrical cavity through a small aperture. Proceedings of the 1989 Electromagnetic Compatibility Symposium, March 1989, Zurich, Switzerland.

[15] NORGARD (J.D.), FROMME (D.C.) and SEGA (R.M.). - Correlation of infrared measurement results of coupled fields in long cylinders with a dual series solution. IEEE-NS Transactions, Vol. NS-37, N6, December 1990, p. 2138-2143. 


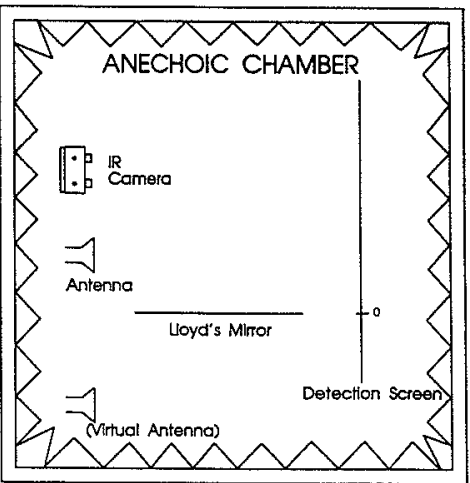

a. Experimental set-up

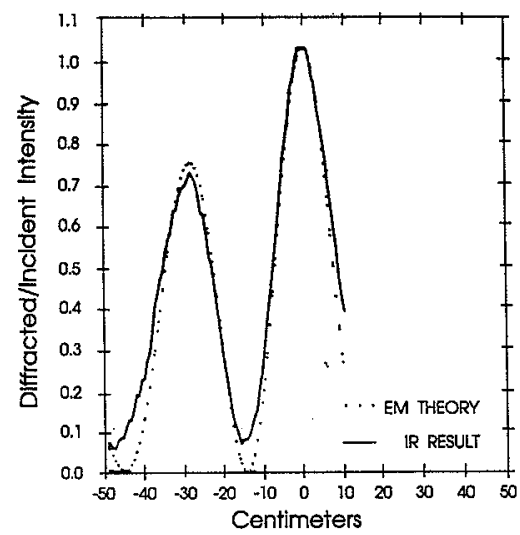

b. Diffraction pattern

Fig. 1. - Lloyd's mirror experiment

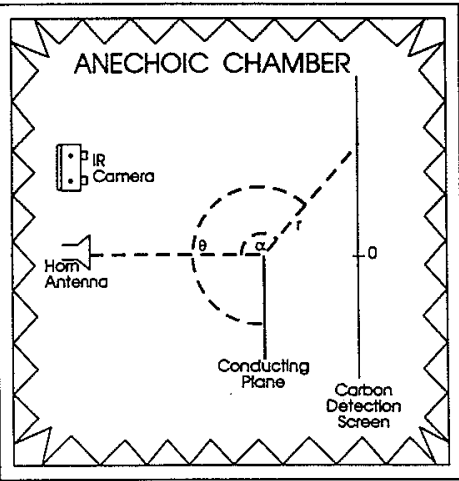

a. Experimental set-up

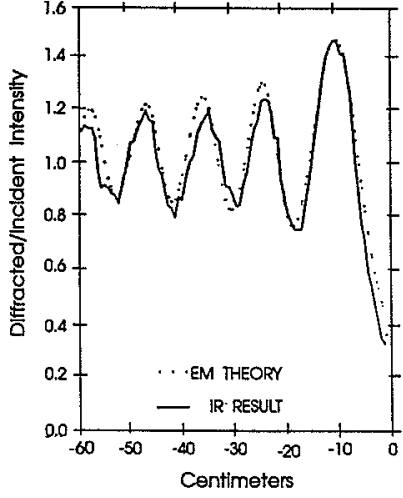

b. Diffraction pattern

Fig. 2. - Conducting half-plane experiment

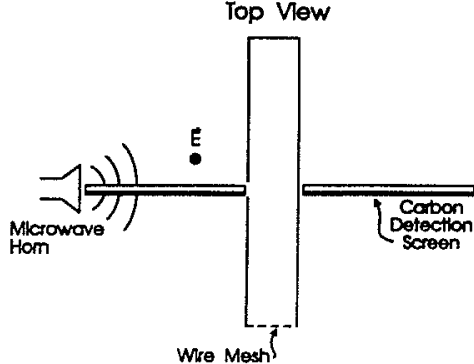

IR Camera

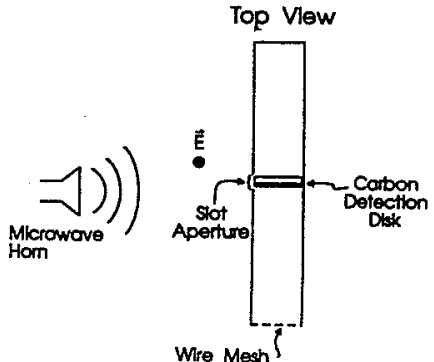

IR Camera

a. Exterior scattered fields

b. Interior cylindrical modes

Fig. 3. - Experimental arrangement 